\title{
La Liga Mexicana Anti-China de Tapachula y la Xenofobia POSREVOLUCIONARIA EN CHIAPAS
}

\author{
The Anti-Chinese Mexican League of Tapachula and the post-revolutionary xenophobia in Chiapas
}

\author{
Miguel Lisbona-Guillén
}

\section{Introducción}

$\mathrm{N}$ el Soconusco es una excepción ni lo fue Chiapas o el mismo Estado mexicano a la hora de generar ciertas manifestaciones xenófobas que tuvieron a los chinos como sujetos de escarnio a través de violencia física o ataques discursivos de muy diversa naturaleza. Desde el vecino país del norte, Estados Unidos, hasta diversos Estados de Sudamérica o el Caribe, la población china fue la protagonista de debates públicos desde el siglo XIX por su exótica presencia como trabajadores que cobraban bajos salarios, esforzados y multifuncionales, tras el fin de la esclavitud. La mano de obra requerida en plantaciones agrícolas, en la construcción de ferrocarriles o en diversos oficios manuales produjo el arribo bajo contrato primero, o de forma libre después, de un buen número de varones chinos, casi todos jóvenes, que huían de los conflictos políticos y bélicos de su país, así como de las deplorables condiciones en las que vivían.

China, el país que más migrantes ha expulsado en el mundo, mandó a muchos de ellos a tierras americanas y algunos recalaron en el Soconusco por distintas vías, aunque en un principio su arribo no fue directo, es decir, la mayoría de los primeros chinos que se

Miguel Lisbona Guillén, Universidad Nacional Autónoma de México. instalaron en Chiapas ya habían tenido experiencias en otros territorios nacionales o extranjeros; sin embargo, una vez asentados y con posibilidades de crecimiento económico hicieron lo que todavía es perceptible en la migración actual: la creación de cadenas. Un familiar traía a otro y éste hacía lo propio con un vecino o un amigo de la localidad de procedencia. La franja costera de Chiapas y la Sierra, e incluso municipios como Jiquipilas y Cintalapa, vieron cómo a finales del siglo XIX, pero sobre todo en las tres primeras décadas del XX, la presencia de trabajadores chinos, reconvertidos en comerciantes de manera acelerada, se hacía visible hasta representar una supuesta competencia para los mercaderes locales, además de ser un aporte de aspectos poco pensables en aquellos años, como la diversificación de productos para la elaboración de alimentos: la reconocida todavía como comida china, bandera identitaria hoy en día de municipios como el de Tapachula.

Muchos son los aspectos que quedan por abordar de esta inmigración a tierras chiapanecas. A pesar de ello, en este texto sólo se presentará y analizará brevemente la creación de la Liga Mexicana AntiChina de Tapachula, rareza desde la perspectiva actual en Chiapas, pero propia de un momento y unas

Recibido: 20 de enero de 2013. 
circunstancias particulares tanto en el país como en el Soconusco.

\section{iHay que mirar al norte! Persecución y creación de organizaciones antichinos}

Según la opinión de Juan Hung, el conflicto laboral en pos de mejores condiciones de trabajo para los chinos en la década de los sesenta del siglo XIX (Hung, 1992: 40), y un movimiento contra los obreros asiáticos en California suscitado en 1875, son el "germen del movimiento antichino, dirigido por elementos racistas en los sindicatos obreros, apoyado por la pequeña empresa y vociferado por demagogos y oportunistas políticos de toda laya." Tales circunstancias provocaron la revisión de los tratados migratorios entre Estados Unidos y China, además de conducir por dos caminos los ataques a los inmigrantes orientales: el primero centrado en la competencia laboral que significaban los migrantes (Hung, 1992: 51-53), y el segundo interesado en crear un discurso degradante sobre la condición humana de los chinos fundamentado en supuestas referencias científicas (Hung, 1992: 40).

El ejemplo, o mal ejemplo, estadounidense ha dado pie a que historiadores como Jean Meyer señalaran, en un breve e inicial trabajo, que lo ocurrido en el país del norte fue la referencia tomada por los mexicanos asentados en los estados fronterizos con Estados Unidos para actuar en su contra en México, hecho discutido con contundencia por diversos investigadores en la actualidad (Reñique, 2003). Pero, sin entrar en estos detalles, es conveniente apuntar que, cuando el régimen porfiriano agonizaba, se encargó desde las instancias gubernamentales, en el año 1903, un estudio para conocer el papel de la migración asiática — china y japonesay discernir si era beneficiosa o perjudicial para el país (Trueba, 1990: 19). Los resultados, basados en dudosas consideraciones raciales en boga en el momento, indicaron que la inmigración debía frenarse y motivaron, incluso, el envío de un inspector a China para discernir las condiciones necesarias que permitieran la debida y correcta inmigración (Trueba, 1990: 19). Incluso antes de la caída de Porfirio Díaz, y anticipando lo que iban a ser las campañas antichinos y los hechos violentos que se produjeron contra los inmigrantes, alguna agrupación política con aires progresistas como el Partido Liberal de los Flores Magón estableció en su Manifiesto a la Nación de 1906 que "La prohibición de la inmigración china es, ante todo, una medida de protección a los trabajadores de otras nacionalidades, principalmente a los mexicanos [...] En general, la inmigración china no produce a México el menor beneficio" (Hernández, 1999: 224).

Su contundencia previene las dificultades que de forma paulatina enfrentaron los chinos al estallar el conflicto armado nacional conocido como Revolución mexicana. Los múltiples frentes de esta confrontación, que avivaron los iniciales resquemores hacia los inmigrantes, tuvieron, con toda seguridad, un punto de referencia nacional e internacional en la matanza de chinos producida en Torreón durante el año 1911 (Puig, 1992). El norte, como se puede comprobar, no se refería únicamente al vecino país, sino también a los estados de la república mexicana. Es en ellos donde florecerá el discurso de la competencia económica que representaban los inmigrantes chinos, competencia que, según se comentaba, se veía reflejada en dos aspectos: uno relacionado con el florecimiento del comercio en manos de estos inmigrantes, y otro que enfatizaba la ocupación de empleos en los negocios de chinos por sus mismos compatriotas, lo que supuestamente impedía a los trabajadores nacionales acceder a ellos.

La familia Elías Calles se ha convertido en referente para conocer la situación que se generó o que permitió la actitud de los gobiernos sonorenses contra los chinos, algo a lo que tampoco fue ajeno Adolfo de la Huerta, quien al ocupar la máxima investidura del Estado quiso expulsar a los residentes asiáticos en 1919 obviando la legislación nacional, parte de la cual era el Tratado de Amistad y Comercio entre China y México (Matute, 1980: 87).

Los años veinte y treinta del pasado siglo se tiñen de arengas que sitúan los problemas económicos de algunos estados en la presencia china, hecho que se verá acrecentado a partir de 1929 con la Gran Depresión norteamericana. Lógicamente, la crisis económica del 
vecino país afectó de manera más ostensible a los estados norteños, pero no fueron los únicos. La repatriación por parte de los Estados Unidos de trabajadores mexicanos desempleados aumentó la presión sobre los temas laborales nacionales, lo que en cierta manera motivó la promulgación de la Ley Federal del Trabajo en 1931. En su artículo noveno, se estableció que los puestos de trabajo correspondientes a trabajadores técnicos y manuales de las empresas con más de cinco personas debían ser ocupados en un $90 \%$ por mexicanos, quedando reducido al 80\% el número cuando el negocio contaba con menos de cinco empleados. Para Pablo Yankelevich esta legislación potenció las campañas antiextranjeras en los lugares que contaban con notable presencia china (Yankelevich, 2004: 21).

Restricciones laborales desde la federación y los estados, y transformaciones legislativas queinvolucraron diversos aspectos de la realidad nacional, pero enfocadas al control de los extranjeros, disminuyeron de manera drástica el ingreso de población china y expulsaron a inmigrantes ya establecidos en tierras mexicanas antes de finales de los años treinta del siglo XX. Lo anterior no impidió, sin embargo, manifestaciones de la opinión pública que todavía insistían en cargar las tintas sobre los males provocados por los asiáticos.

Lasinstituciones en los gobiernos posrevolucionarios tuvieron actuaciones ambiguas; por una parte, secundaron acciones contra los chinos a través de decretos y leyes o las reformas a los mismos y, por otra, intentaron mantener las relaciones con China llamando la atención a las autoridades locales cuando los excesos cometidos en contra de los inmigrantes alcanzaban niveles internacionales, aunque realmente nunca actuaron con decidida convicción, al menos en el caso de los estados del norte. También participaron de forma individual los funcionarios al amparo de las instituciones que representaban, dando soporte a las campañas, comités o ligas que se crearon para dar una institucionalidad a movimientos que fueron conformados por diversos grupos de ciudadanos mexicanos.

José Ángel Espinoza, destacado antichino en los años treinta del siglo pasado, redactó al final de una de sus obras xenófobas un proyecto de Acta Constitutiva para la creación de Ligas Nacionalistas (Espinoza, 1931: 233-238), además de incluir un Proyecto de Estatutos para el Régimen y Funcionamiento de las Ligas Nacionalistas de la República (Espinoza, 1931: 239-274), puesto que su iniciativa no estaba pensada para Sonora o los estados del norte, sino que debía abarcar todo el país.

Sin embargo, se puede afirmar que al iniciar los años veinte del siglo XX ya existían diversos comités antichinos en los estados del norte, periodo coincidente con la finalización del gobierno de Álvaro Obregón -1920-1924- y el inicio del de Plutarco Elías Calles -1924-1928 - (Monteón y Trueba, 1988: 81-83). Es decir, la presencia de comités antichinos en los años veinte estaba secundada, en la década de los treinta del siglo XX, por el surgimiento de diversas organizaciones en la capital del país. Es así como en 1930 surgen el Comité Nacional Pro-Raza, la Unión de Comerciantes Mexicanos, la Liga Mexicana Anti-China y Anti-Judía, la Juventud Nacionalista Mexicana o la Legión Mexicana de Defensa (Gojman, 2000: 174-175). Fue en esos años cuando en distintos estados de la República aparecieron organizaciones que tenían en el nacionalismo exacerbado y xenófobo su fundamento, adornado con referencias a la competencia laboral de los chinos, la lucha por la higiene social o el temor a la degeneración proveniente de un mal mestizaje.

\section{¿Qué ocurrió en Chiapas?}

Lo sucedido en el país también se observó en el estado de Chiapas. La efervescencia nacionalista no tardó en verse reflejada en el municipio que contaba con mayor número de inmigrantes chinos: Tapachula. Por tal motivo, el ll de octubre de 1930 se constituyó la Liga Mexicana Anti-China en dicha ciudad. Su registro en la presidencia municipal y el acta constitutiva de esta asociación, conformada por "50 ciudadanos mexicanos por nacimiento," la convirtió en una organización visible, además de afiliada a las ligas y comités similares de la República. Sus ideales eran similares a los de otras agrupaciones coetáneas que fueron incluso, como sus mismos fundadores afirmaron, su inspiración. No 
parece que el ejemplo cundiera de forma inmediata en la geografía chiapaneca, aunque se tiene constancia de la existencia de una Liga Nacionalista Pro-Raza en Arriaga en 1932, y de otra en Tonalá en el año 1938 (Lisbona, en prensa).

La Liga Mexicana Anti-China en Tapachula ocupó una casa en la ciudad costeña y desde ella intentó distintas estrategias para transmitir sus ideales o dificultar el trabajo cotidiano de los inmigrantes. Manifestaciones, ciertos ataques a comercios y, sobre todo, campañas públicas en los medios de comunicación locales o mediante panfletos, fueron muy comunes hasta su disolución. Uno de los documentos que en estas páginas se presenta responde a este último tipo de estrategia. Al igual que otros que surgieron en diversos estados del país, en su contenido se concentran los estereotipos manejados por dichas agrupaciones, en nada ajenos a lo expresado por la prensa nacional o por políticos antichinos para referirse a tales inmigrantes. El control económico ejercido en ciertos rubros, la no contratación o el maltrato a los trabajadores mexicanos, la transmisión endémica de enfermedades, los peligros que significaban para el mestizaje - ligado todo ello a la concepción racial que se tenía de ellos-, o su inclinación a los vicios de todo tipo son definiciones recurrentes en el periodo más álgido de las campañas antichinos.

Las acciones de estos grupos se encuentran documentadas, también, en las protestas de los afectados, los chinos, o gracias a los representantes legales del país asiático en México, así como en la actitud tomada por los gobiernos estatales en turno. Un ejemplo del primero de estos casos es el segundo documento que ilustra este texto. Los inmigrantes chinos, que se habían organizado paulatinamente en distintas agrupaciones, básicamente en el Kuo Ming Tang o Partido Nacionalista Chino y en la Cámara de Comercio y Agricultura China, asumirán su defensa frente a los ataques denunciándolos a las autoridades locales, estatales y nacionales. Es decir, en los inicios de la Liga Mexicana Anti-China de Tapachula, representantes de los pobladores chinos, como el máximo dirigente de la Cámara China de Comercio y
Agricultura en Tapachula, Samuel Juan, usarán todos sus recursos para contrarrestar lo que veían como una agresión similar a la sufrida en los estados del norte del país. Normalmente, este tipo de solicitudes se fundamentaba en los derechos que tanto los ciudadanos mexicanos - hay que recordar que algunos chinos ya se habían nacionalizado-, como los extranjeros, tenían en territorio de la República. El apelar a la legislación vigente o a los derechos ciudadanos fue un recurso utilizado constantemente por los inmigrantes asiáticos, y más cuando, como en el caso ejemplificado, la carta dirigida al presidente de la República está signada un mes después de conformarse la Liga Mexicana AntiChina en Tapachula.

Aunque la violencia o los furores antichinos no fueron en Chiapas de la magnitud de los vividos en los estados norteños del país, ello no significa que no existieran. Tales circunstancias son difíciles de medir cuando se trata de vidas humanas, y la actual presencia de muchos descendientes de chinos en el estado, sobre todo en la costa chiapaneca, no impide señalar que existen localidades en las que se produjeron hechos violentos y donde los descendientes de aquellos migrantes no habitan como, por ejemplo, el municipio de Villa Comaltitlán.

Con respecto a lo ocurrido en aquellos años, y que finalizó con la desaparición de las agrupaciones antichinos, es pertinente apuntar que la contradicción fue la manera de tratar el tema de los inmigrados desde los gobiernos estatales. Gómez Izquierdo señaló que la publicación en junio de 1929 de un alegato antichino en El Heraldo de Huixtla, reproducción de un artículo aparecido en otros periódicos del país, motivó la queja de la legación china ante las autoridades de la Secretaría de Relaciones Exteriores. El resultado de la reclamación fue la reacción de las autoridades judiciales del estado suprimiendo la publicación del periódico (Gómez, 1991: 157). Lo anterior, tomado como síntoma de lo ocurrido en Chiapas por dicho autor, ejemplifica una actuación frente a las campañas xenófobas, pero no resume la diversidad de circunstancias vividas.

Los poderes locales, como los estatales, tuvieron acciones ambiguas, ora acercándose a los gobernantes 
del norte de México y de la Federación claramente antichinos, ora asumiendo lo pretendido por la Secretaría de Relaciones Exteriores de México, más propensa a disipar el clima agresivo ante los inmigrantes. Es decir, acierta Gómez Izquierdo al afirmar que el gobierno de Raymundo E. Enríquez y algunas autoridades locales se mostraron, después de 1930, reacios a secundar las campañas antichinos, pero ello no implica que las dejaran de asumir cuando estaba en juego la relación con la Federación y, en concreto, con el presidente de la República en turno y sus subordinados. No es lo preciso de las acciones lo que caracterizó el papel gubernamental en Chiapas, sino que éstas parecen tomar un rumbo más claro a partir de la formación de las agrupaciones antichinos en territorio chiapaneco.

Lo anterior se observa en las quejas de los miembros de estas agrupaciones contra el comportamiento de las autoridades locales, situación que llegó a un momento culminante cuando la presidencia municipal de Tapachula acordó retirar los rótulos de la Liga Mexicana Anti-China del local que ocupaba y amenazó a sus miembros de consignación si seguían con la campaña de hostigamiento agresivo hacia los asiáticos (Lisbona, en prensa). A pesar de estas circunstancias, el acoso organizado contra los chinos continuó, aunque de una forma más velada, incluso después de desaparecida la Liga Anti-China de Tapachula en el año 1935 (Gómez, 1991: 158).

El resultado de las campañas antichinos y del clima de violencia visible o solapada fue una notable reducción de la población china, según los datos censales de los años cuarenta del siglo XX. Sin embargo, aquellos que se asentaron en el territorio de Chiapas construyeron, a través de acciones individuales o mediante las ya constituidas organizaciones chinas, formas de relación e inclusión en la sociedad de acogida visibles hasta el presente.

\section{Bibliografía}

Espinoza, José Ángel (1931), El problema chino en México, México: Porrúa.

Gojman de Backal, Alicia (2000), Camisas, escudos y desfiles militares. Los Dorados y el antisemitismo en México (19341940), México: UNAM/FCE.

Gómez Izquierdo, José Jorge (1991), El movimiento antichino en México (1871-1934). Problemas del racismo y del nacionalismo durante la Revolución Mexicana, México: INAH.

Hernández Padilla, Salvador (1999), El magonismo: historia de una pasión libertaria, 1900-1922, México: Era.

Hung Hui, Juan (1992), Chinosen América, Madrid: Mapfre. Lisbona Guillén, Miguel (en prensa), Allí donde lleguen las olas del mar... Pasado y presente de los chinos en Chiapas, México: UNAM.

Matute, Álvaro (1980), Historia de la Revolución Mexicana, 1917-1924. Las dificultades del nuevo Estado, vol. VII, México: COLMEX.

Monteón González, Humberto y José Luis Trueba Lara (1988), Chinos y antichinos en México. Documentos para su estudio, México: Unidad Editorial/Gobierno de Jalisco.

Puig, Juan (1992), Entre el río Perlas y el Nazas. La China decimonónica y sus braceros emigrantes, la colonia china de Torreón y la matanza de 1911, México: CONACULTA.

Reñique, Gerardo (2003), "Región, raza y nación en el antichinismo sonorense”, en Aarón Grageda (coord.), Seis expulsiones y un adiós. Despojos y expulsiones en Sonora, México: UNISON/Plaza y Valdés, pp. 231-289.

Trueba Lara, José Luis (1990), Los chinos en Sonora: una historia olvidada, Hermosillo: Universidad de Sonora.

Yankelevich, Pablo (2004), "Nación y extranjería en el México revolucionario", en Cuicuilco, mayo-agosto, vol. XI, núm. 31, pp. 1-29. 


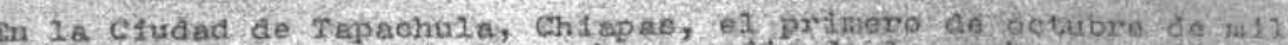

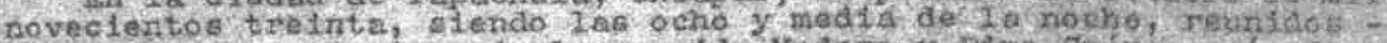

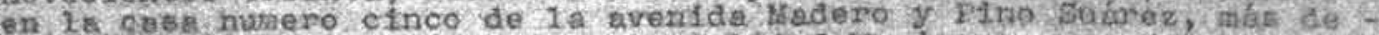

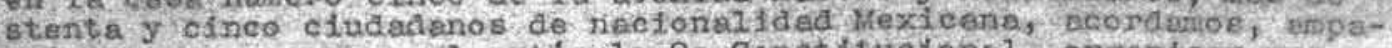
rados $y$ con apoyo an el axtílculo 9o.Constituclonial, orzantzax una agmapeción Anti-China, bajo el siguiente Probrama de Acctón

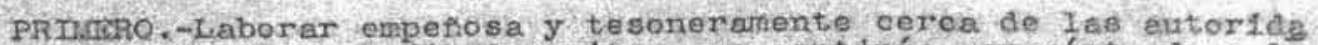
des coupetentes, con el In de ovitar que continúe acrecerntendose la invasión de 108 lndividuos de raza china, tanto al pais en general como al Setado de Chiapas y al Departamento de Soconusco en 10 part 1 cular.

SEGUNDO,-Trabajor con ahinco, por todos $10 s$ medios legales, para evitar que se abran al público nuevos estabiecinientos comerciarles de propiedad o regenteados por individuos de la nacionalidad antes mencionada.

TERCFRO.-Gestionar ante quien corresponda todo io conducente hasta obtener que se observe estricta y escrupulosamente lo estatuldo en nuestra Carta Fundamental en 20 que respecta a la apilcación de Ias Leyes de Inmigración, esi como de 1 as que se aponen terminante y radicalmente a la adquisicion de bienes raices por extranjeros no nacionalizados mexicanos.

CUARTO.-Pedir a la H. Cámara de Diputados la elaboración, $y$ promulgación y cumplimiento al Primer Mandatario de la Nación, de -una Ley que impida la concestón ge la nacionalidad y ciudadania mexl cana a todo individuo de nacionalldad china, sea cual fuere el tiempo de permanencia en el pafs; y el retiro inmediato de tal beneficio a los que actualmente estén disfmutando de 61 .

QUINTO.-Exijir a quien corresponda que todos los comercios -chinos establecidos, sin excepción de ninguna naturaleza, cumplan -.. exactamente los mandamientos de las Leyes vigentes en materia de trabajo.

SEXTC.-Gestionar con deciaión y empefio, que se lleve a cabo el anhelo de todo mexicano de que se confine a todos los individuosde nacionalida china, $a$ un barrio separado y distante de los centros comerciales, tres kilometros por lo menos, cuyo barrio sera delinitado perfectamente por quien corresponda, y en el cual no podrán vivir, bajo severisimas sancfones, mujeres de nacionalidad mexicana, asi como los individuos chinos estaran absolutamente vedados de expandirse más allá del barrio aludido.

SEPTINO.-Firmemente convencidos de que el más pesado lastre que implae el progreso y elorecimiento de Méxíco, lo constituye la rg za china que nos ha invadido totalmente, los suscritos protestan que todos y cade uno de sus actos propenderan a obtener 1 a DESCHINATIZA-CION DDI, ESTADO Y DEL DEPARTANDNTO para cuyos fines se harán propagan das perídica y sistemáticamente en cada uno de los pueblos de esta Eritidad.

OCTAVO.-Todos Ios asuntos de 1a agmupación, que se acorab denondnar "LIGA MEXTCANA ANTI-CHINA" serán tratados por el PNestant. y Secretario de la misma, o por falta o ausencia de estos, porios.demás miembros de la misma, en el grado que corpespotida y pepacuerá en asamblea. 


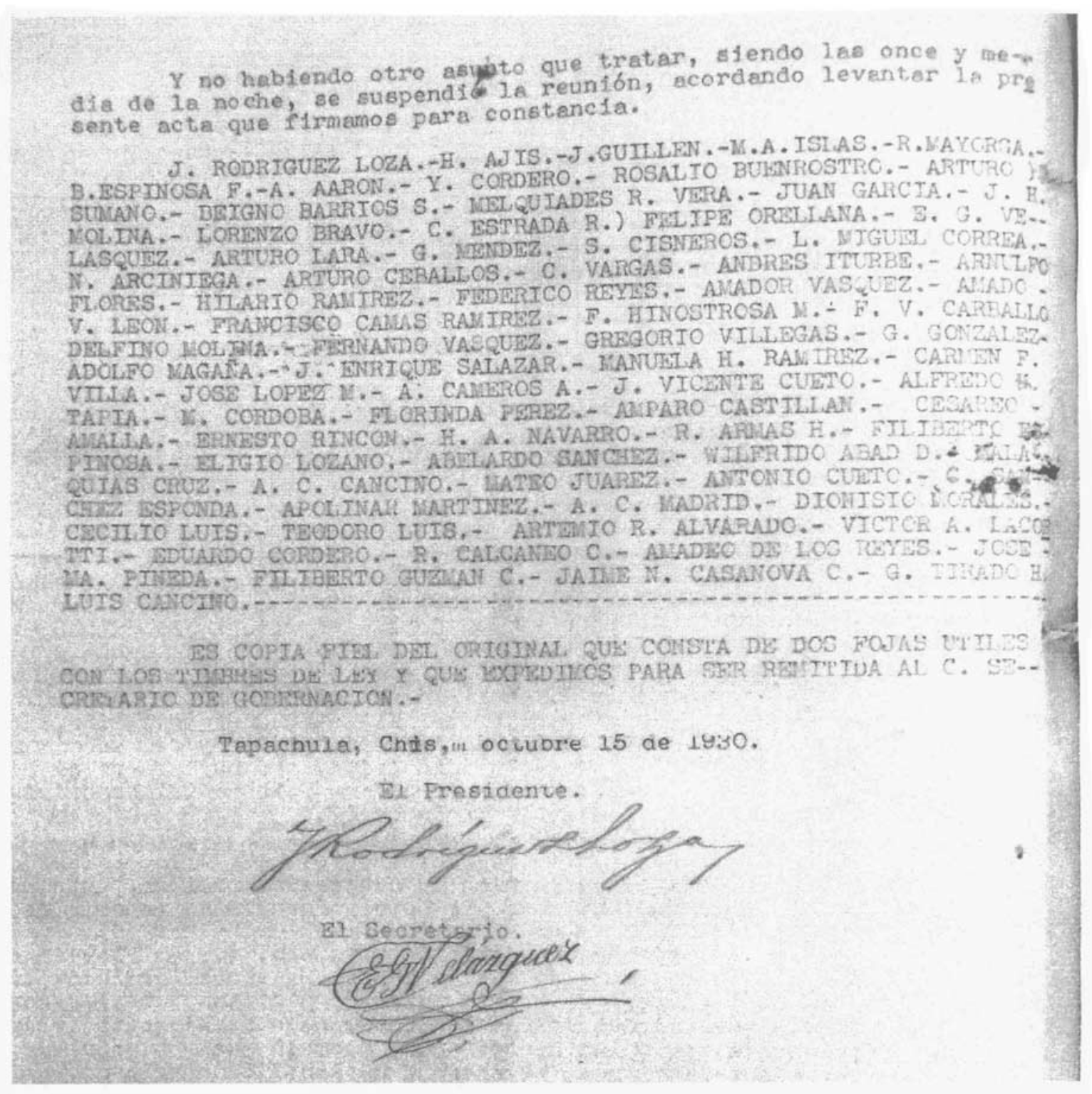




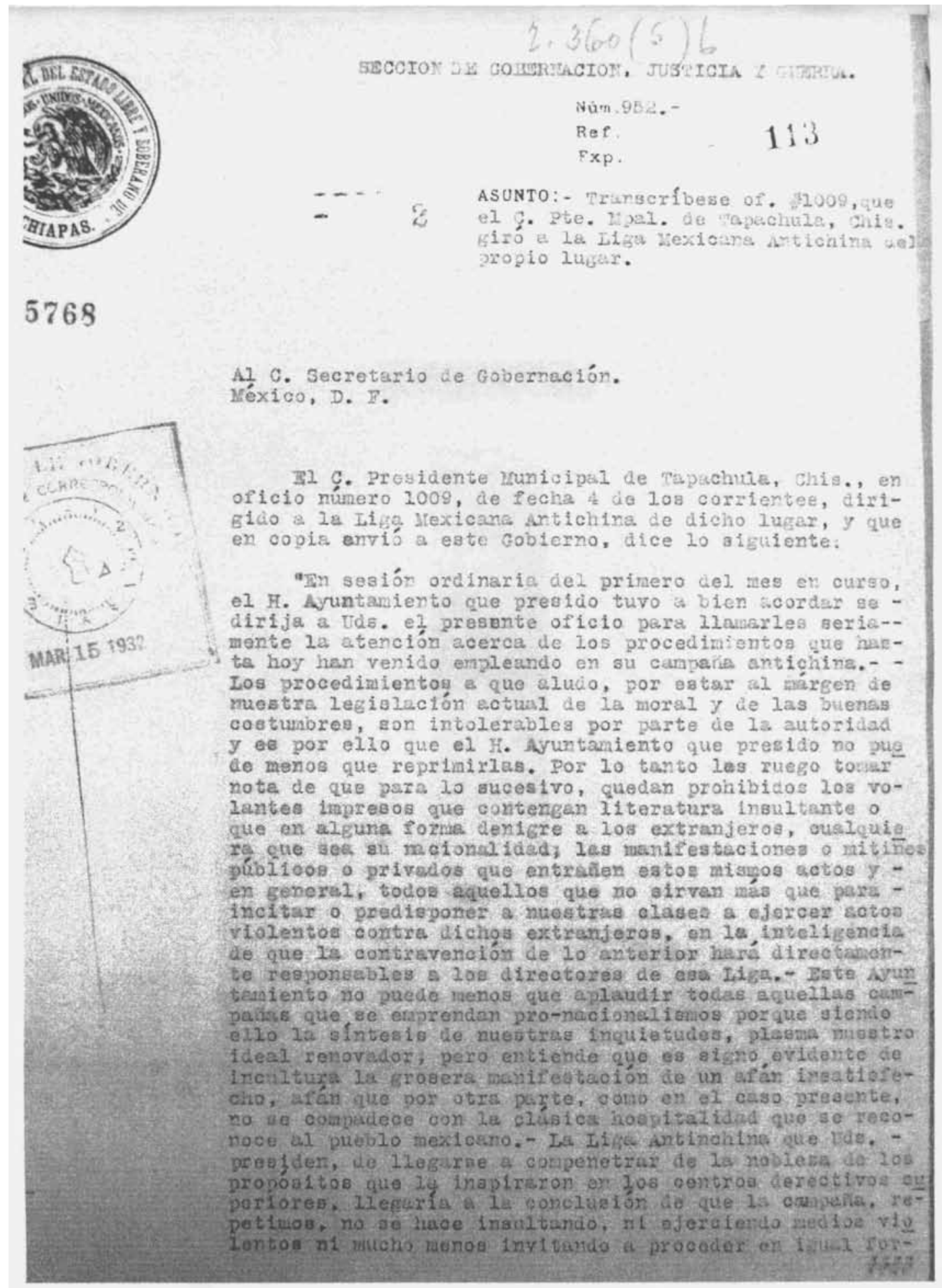




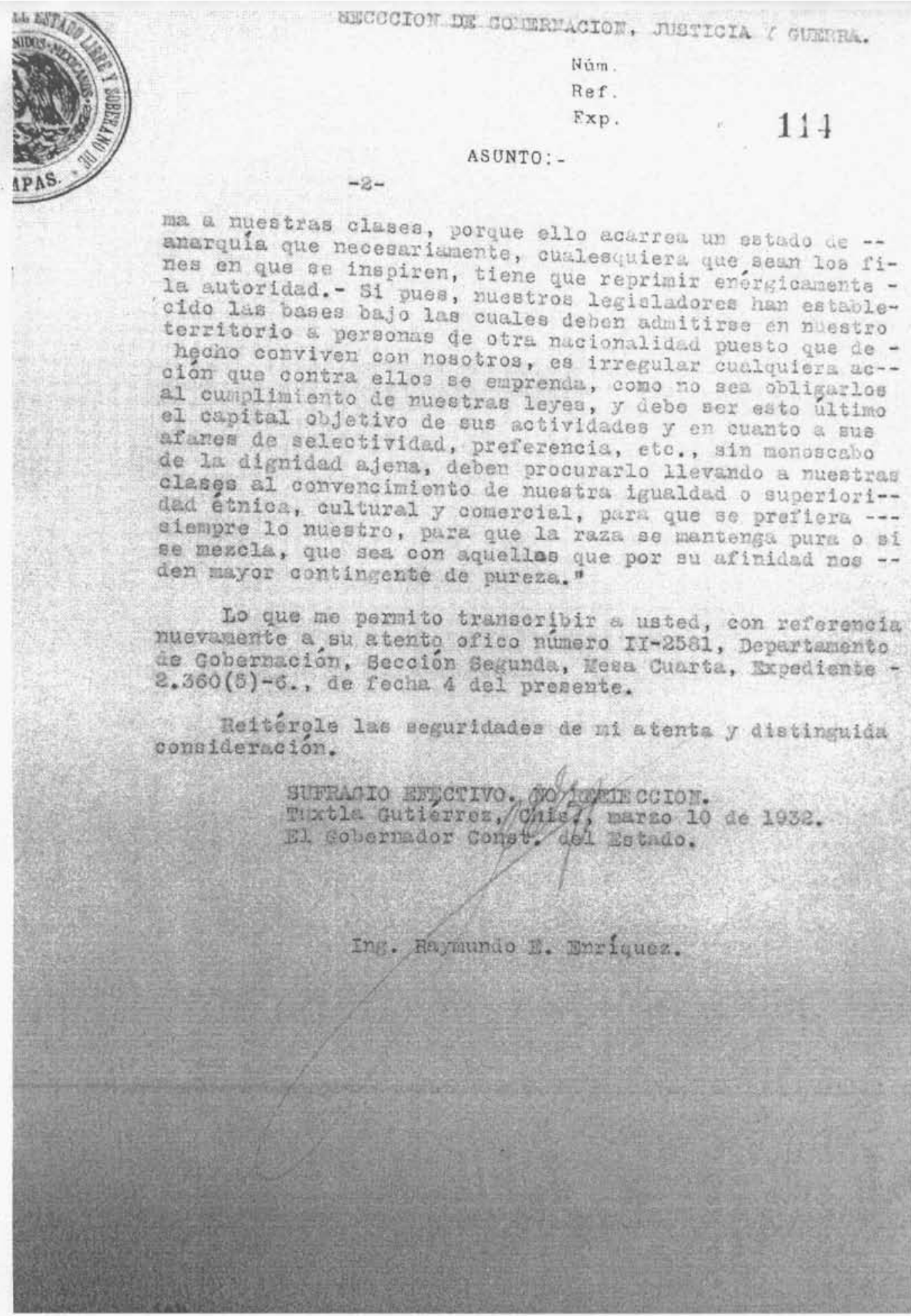

